\title{
The Beauty of Consistency in Radio-Scheduling Decisions
}

\author{
Eirini Liotou*, Raimund Schatz*, Andreas Sack1*, Pedro Casas, Dimitris Tsolkas*, Nikos Passas*, Lazaros Merakos* \\ *Department of Informatics \& Telecommunications, University of Athens, Greece \\ ¥AIT Austrian Institute of Technology, Austria \\ * (eliotou, dtsolkas, passas, merakos\}@di.uoa.gr \\ *\{forename.surname\}@ait.ac.at
}

\begin{abstract}
Radio scheduling is a well-studied problem that has challenged researchers throughout the last decades. However, recent findings that stem from the Quality of Experience (QoE) domain come to give a new perspective to traditional radio scheduling approaches. In this paper, we take advantage of recent subjective results regarding the impact of throughput fluctuations on the QoE of interactive applications and revisit well-known scheduling algorithms. By quantifying the impact of traditional radio schedulers on user-perceived QoE, we manage to draw new conclusions regarding the radio scheduling problem, such as the importance and impact of consistency of the resource allocation decisions on the users' QoE. As main result, fair algorithms inherently seem to be more consistent than greedy ones, providing less throughput fluctuations and, thus, better QoE. Based on this outcome, we propose a new scheduling approach, which further improves end-users' QoE by moderating throughput fluctuations.
\end{abstract}

Keywords-QoE management, QoE models, KPI, throughput fluctuations, radio schedulers, fairness, consistent user experience.

\section{INTRODUCTION}

Nowadays, the Quality of Service (QoS) and the resulting Quality of Experience (QoE) of mobile end-users keeps improving thanks to the development and roll-out of new network technologies and standards. In this context we witness a trend towards rising importance of a new quality criterion: network stability in terms of consistent performance experienced by the end-user. This prioritization is visible in the recently published NGMN 5G Whitepaper [1] in which "consistent user experience" forms an integral part of the $5 \mathrm{G}$ vision. This is not surprising, since the trend towards ever rising peak rates (as enabled by new Radio Access Network (RAN) technologies) also increases the probability of wireless users experiencing larger network performance fluctuations. Moreover, due to the diversity in the RAN technologies and the heterogeneity within the cellular infrastructure (e.g. overlaying femtos, etc.), the phenomenon of throughput fluctuations becomes even more intense.

Furthermore, fluctuations have a noticeable impact on subscribers' QoE. In [2], we have demonstrated on behalf of subjective user testing results that throughput fluctuations have a significant negative impact on the end-user experience. Focusing on interactive networked applications, we suggest that novel downlink-throughput related Key Performance Indicators (KPIs) have to be developed for proper QoE-based traffic analysis in mobile networks. For the domain of QoEbased network management these results imply that avoiding throughput-related quality fluctuations leads to significant QoE gains.

In general, consistency (in terms of fluctuation avoidance) can be achieved using two different strategies: a) by mitigating the application-level impact of throughput fluctuations, or b) by smoothing throughput on the network-level itself. Each one of these strategies corresponds to a different business case.

On the one hand, application-level strategies are driven by Over-The-Top (OTT) players, who have the means and the interest to control their customers' $\mathrm{QoE}$ by handling application level parameters that they can control. A prominent example is HTTP Adaptive Streaming (HAS), which in essence dynamically changes the media quality (or bitrate) of video segments requested in order to avoid playout buffer starvation. In the HAS context, switches among different layers (i.e. "fluctuations" of media quality) have been identified as an important QoE influence factor (e.g. [3]), and thus video adaptation algorithms with a smoothness logic have been proposed (e.g. [4]).

On the other hand, network-level strategies are driven by Mobile Network Operators (MNO), who only have the means to control the QoE of their customers through lower-layer parameters. For instance, network-aware bit-rate adaptation schemes have been proposed for UDP/RTP-based streaming (e.g. [5]), as well as joint rate adaptation and admission control schemes that control how long and by how much the predicted video quality fluctuates/falls below a certain threshold [6].

Future business models even describe the collaboration of OTTs and MNOs, where cross-layer approaches can be envisioned. For instance, [7] proposes a way to mitigate temporal quality fluctuations using lower layer information (e.g. channel quality) and application layer information (e.g. application utility in term of MOS).

In this paper, motivated by the MNOs' need to provide good QoE for their customers, without relying on OTT players to achieve that, we give our focus on the second strategy, namely on network-level fluctuation mitigation. By giving a solution at network level, we do not depend on different application implementations to solve the same problem, but rather provide a catholic and centralized solution to the MNO's interest (i.e., an application- and device-independent solution).

One promising network-level QoE management approach that can help increase $\mathrm{QoE}$ is to ensure stable amounts of 
bandwidth available to each user. In this context, schedulers play a central role as they directly influence the radio resource allocation per user. This paper adopts this technique, and quantifies the impact of radio scheduler behavior on QoE. More specifically, the paper's contribution lies in

a) evaluating current state of the art schedulers regarding the throughput fluctuations they cause and the respective QoE performance based on fluctuations-aware KPIs, and

b) proposing an inherently fluctuations-avoiding scheduler, that further improves the QoE of end-users.

Our proposal mainly concerns real-time interactive applications, namely web browsing, google maps, IPTV, video-conferencing, etc., where fluctuations are mostly observable; but the implementation itself is applicationunaware (so OTT-cooperation is not required). This way we help MNOs better understand and improve the experience of their customers without relying on other parties.

The remainder of the paper is organized as follows: In section II, QoE models and metrics are provided that can be useful to evaluate QoE in throughput fluctuation situations. In section III, the background on traditional radio schedulers is given, explaining their design objectives. Using the said QoE models, scheduling algorithms are compared in terms of QoE in section IV, while the importance of accounting for fluctuations when designing new radio schedulers is revealed. Section $\mathrm{V}$ describes a novel radio scheduler that inherently accounts for throughput fluctuations, while Section VI evaluates it. Our conclusions are presented in section VII.

\section{QoE Models AND Metrics to Evaluate QoE}

When discussing throughput fluctuations we have to distinguish between two different cases: (i) firstly, throughput may fluctuate as a consequence of the normal behavior of an application and/or the natural usage pattern of the end user; for example, the YouTube downlink throughput presents a very clear on/off fluctuation pattern as a consequence of the chunkbased flow control of the application, and the downlink throughput pattern of a web browsing session is highly dependent on how fast a user browses a site and goes to the next one. In the second case, (ii) throughput fluctuates as a consequence of variations in the bandwidth of the corresponding network connection. In mobile networks, the bandwidth of a connection can vary for multiple and very different reasons, such as fast and slow fading, interference, changes in coding and modulation scheme, scheduler algorithm, resource constraints, contention with other users, handovers, etc. As also implied in the introduction, here we focus on this second case, which represents the undesirable and uncontrollable fluctuations stemming from the network.

In [2] we have presented a complete study of the QoE undergone by 52 mobile users in controlled subjective lab tests, using different mobile applications such as YouTube, Web browsing and Google Maps. Their results suggest that novel downlink throughput related KPIs must be defined for QoE-based traffic analysis in mobile networks. The common approach to consider only average throughput values is insufficient to describe subjectively perceived network quality in the case of news site browsing and browsing Google Maps. Whereas a constant bandwidth of $2 \mathrm{Mbit} / \mathrm{s}$ for browsing a Google Map led to a Mean Opinion Score (MOS) of $\approx 4$, an alternating bandwidth of 0 and $4 \mathrm{Mbit} / \mathrm{s}$ (average throughput is also $2 \mathrm{Mbit} / \mathrm{s}$ !) led to a MOS of $\approx 2.6$ (see Figure 1 ). For the case of YouTube, the difference between the two MOS values (constant bandwidth vs. fluctuating bandwidth but identic average throughput) is even bigger: MOS of $\approx 4.5 \mathrm{vs}$. MOS of $\approx 2.5[2]$.

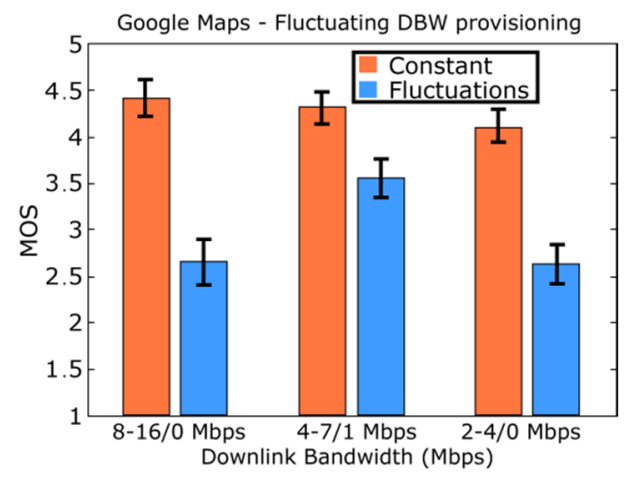

Figure 1: Subjective QoE results for Google Maps browsing, with constant and fluctuating bandwidth of the same mean value [2].

Hence, in [2] a first approach is presented how to define fluctuation-specific KPIs by considering the amount of time in which the throughput is below a certain throughput threshold. With this approach, the so called Effective Average Download Throughput (EADT) can be determined and utilized to calculate the realistic MOS value by multiplying the plain Average Download Throughput (ADT) by a model-dependent Correcting Factor (CF), namely:

$$
Q o E=f(E A D T), \text { where } E A D T=C F * A D T .
$$

Based on the previous work, in [8] we discuss and evaluate five models to derive the EADT. In the first one (LTD, LowThroughput Duration), the CF is determined by the fraction of time that the throughput is below a certain downlink bandwidth threshold. The second model (SLTD, Selective Low-Throughput Duration) is similar to the first one, but it assumes that short time bandwidth drops are not perceived by the users. Instead of using a fixed download bandwidth throughput, the third model (TJ, Throughput Jitter) uses a moving average-based threshold e.g. a sliding window length of 5 seconds. The fourth model (AREA, Area-based model) does not only consider the time below a threshold, but also accounts for how deep the corresponding throughput gap is. The fifth model (DOUBLE) is similar to LTD but considers two different bandwidth thresholds. For more detailed information about the presented models please see [8].

In the same work, we have also evaluated these models via empirical user studies. Obviously, the optimal model depends on the scenario (browsing Google Maps, News Site, etc.) and the specific fluctuation pattern (progressive outages with disconnections and subsequent recoveries vs. fast bandwidth changing environments vs. high/low bandwidth profile with fast short-scale variations). Overall, we proposed a set of first generic throughput fluctuation models that allow for quantifying the impact of throughput fluctuations on QoE. 


\section{BACKGROUND ON RADIO SCHEDULING}

\section{A. Traditional objectives of scheduling algorithms}

Radio scheduling is the problem of allocating spectrum resources to competing user requests. Since, commonly, these requests exceed the number of available resources, intelligent radio schedulers need to be designed. Radio schedulers, as of today, are designed to meet four objectives [9]:

Increase spectral efficiency: This objective guarantees the efficient utilization of the radio spectrum, commonly expressed in bit/s/Hz. This can be achieved by accounting for the channel conditions between the base station and the various users in a cell, while taking scheduling decisions. As a consequence, users with better channel conditions get more spectrum resources and hence, achieve higher data rates. In this way, the sum cell throughput is also increased.

Increase fairness: If spectral efficiency was the only criterion for radio scheduling, users with bad channel conditions (e.g. at cell edge) would starve. Therefore, fairness guarantees that even those users receive a decent service in the long run.

Satisfy QoS guarantees: Different flows may have different QoS requirements and constraints, such as a minimum Guaranteed Bit Rates (GBR), maximum acceptable packet delays, etc. QoS-specific schedulers have been designed in order to respect such special requirements.

Achieve low complexity and good scalability: This requirement guarantees that scheduling decisions can be actually taken in real-time, so that they can be implemented into a real base station.

In the literature, a plethora of proposed schedulers can be found that take into account the previous factors. Since, however, these four objectives actually compete with each other (e.g. spectral efficiency vs. fairness, QoS guarantees vs. complexity, etc.), trade-offs need to be made in their design.

\section{B. State of the art scheduling algorithms}

Radio scheduling is the problem of allocating $K$ resources to $N$ users. Its solution is based on estimating a "priority weight" or "metric" in favor of allocating resource $k$ to user $j$. A comparison of these weights leads to the decision about which resource will be allocated to which user. The rule is that resource $k$ is allocated to user $j$ among all users $i$, if the following metric is the highest one, namely:

$$
m_{j, k}=\max _{i}\left\{m_{i, k}\right\} \forall i=1 . . N, k=1 . . K
$$

One scheduling decision is taken per available resource (i.e. per spectrum unit) per Transmission Time Interval (TTI) and per base station. Some of the most popular scheduling algorithms are presented below:

Resource Fair (RF) or Blind Equal Throughput (BET): The metric that is estimated by this scheduler is the following:

$$
m_{i, k}=\frac{1}{r^{i}(t)}
$$

where $r^{i}(t)$ is the achieved data rate at current time $t$ due to the resources already allocated to user $i$ during the same TTI. Thus, the only objective of this scheduler is to achieve fairness in the resource distribution. It is worth noting that the current decision of this scheduler depends on its previous decisions.

Maximum Throughput (MT): The metric used in this case is the potentially achieved data rate by each user, if this user is indeed scheduled with the examined resource, namely:

$$
m_{i, k}=d_{k}^{i}(t)
$$

where $d_{k}^{i}(t)$ is the expected data rate when assigning resource $k$ to user $i$. This expectation relies on feedback from the users to the base station about the experienced channel conditions. Therefore, according to this metric, users with better channel conditions will get more resources, since they will be able to take better advantage of the channel and support the reception of more bits per second (Downlink). It is interesting, that this scheduler depends on current channel estimations only.

Proportional Fair (PF): This scheduler is a compromise between the previous two, and is widely used today:

$$
m_{i, k}=\frac{d_{k}^{i}(t)}{r^{i}(t)}
$$

The Proportional Fair scheduler tries to find a balance between spectral efficiency (i.e. maximum throughput in the system) and fairness among the users.

As regards the RF and PF schedulers, a "fairness window" in the past can be also considered, in which case fairness is targeted over a longer time window. In this case, predefined weights are given to the past window and current timeframe.

Towards a new "consistency" objective: It becomes evident that, throughput fluctuations are not considered by these state of the art schedulers (or their variations, thereof). Taking, however, into account that throughput fluctuations directly affect QoE, we here introduce a fifth, new objective for the radio schedulers' design i.e., a "consistency factor". This new objective may be added to the list of the four objectives presented above (in section III-A). In this context, we provide the following definition:

Definition: A scheduler is characterized as "consistent" if it minimizes the occurrence or the altitude of throughput fluctuations. This may be possible for instance by providing highly constant available bandwidth levels to each user.

Next, we are going to investigate how traditional state of the art schedulers perform from a QoE-perspective, using a selection of the models introduced in Section II.

\section{COMPARISON OF TRADITIONAL SCHEDULERS}

\section{A. Fluctuations-specific comparison}

As elaborated before, fluctuations play a crucial role in the perceived QoE. However, existing scheduling algorithms have not been designed with this in mind, and thus, their impact on QoE is unknown. The purpose of this section is therefore to compare current schedulers based on the QoE models described in Section II and to draw conclusions regarding their efficiency into mitigating throughput fluctuations.

The evaluation of these algorithms has been performed using the LTE-A Downlink System Level Simulator (v1.8 r1375) [10], using the input parameters of Table 1. In LTE-A the scheduling interval (TTI) corresponds to $1 \mathrm{~ms}$. 

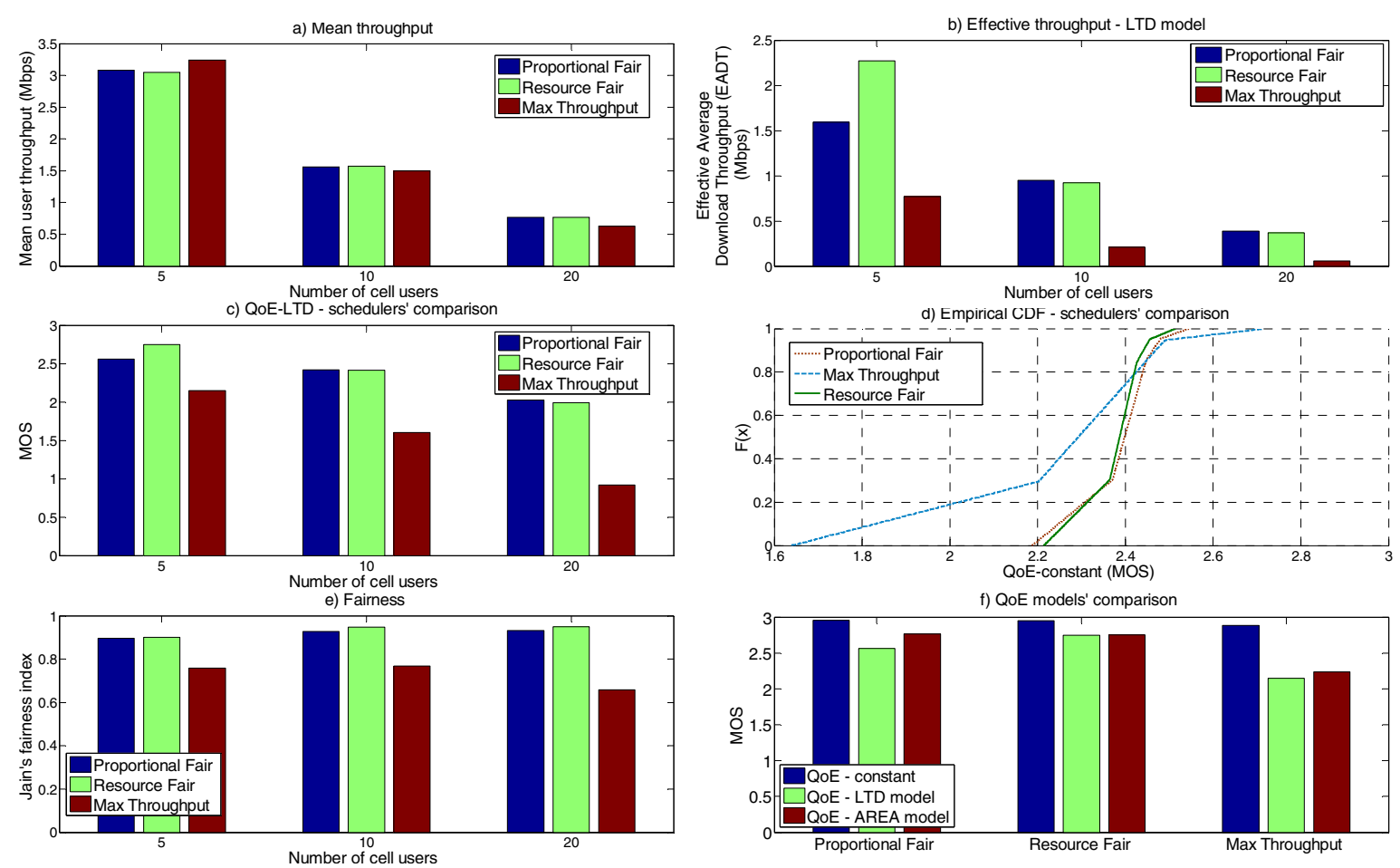

Figure 3: Comparison of state of the art schedulers: a) Average Download Throughput, b) Effective Average Download Throughput - LTD model, c) QoE - LTD model, d) CDF of QoE scores (for 20 users), e) Fairness, f) QoE models (for 5 users).

Figure 2 below shows the instantaneous experienced throughput at a random LTE-A user during 10s. Throughput values are smoothed over a $50 \mathrm{~ms}$ window instead of being presented for each scheduling interval of $1 \mathrm{~ms}$, for higher readability. The observed fluctuations are a result of the uncontrollable, instantaneous channel conditions, but also of the scheduling algorithm decisions. Since, however, we have used the same channel conditions across all schedulers in this experiment, the resulting differences in the fluctuations' magnitude are caused solely by the scheduling algorithms themselves. This shows that the selection of the scheduler has a strong influence on the resulting fluctuations.

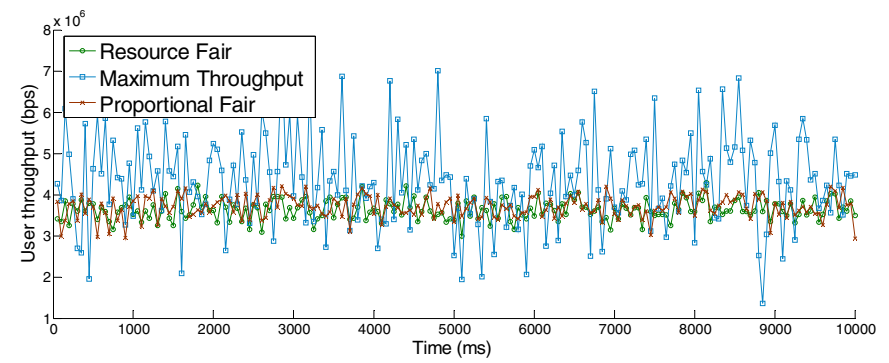

Figure 2: Fluctuations experienced by a random LTE-A user by the three state of the art schedulers.

Regarding the fluctuations' impact of each scheduler, it is shown in Figure 2 that the Proportional Fair and Resource Fair schedulers lead to lower throughput fluctuations. The Maximum Throughput scheduler, on the contrary, leads to significant fluctuations. Therefore, there seems to exist some correlation between the number/magnitude of fluctuations and the fairness of the scheduler. That is, the fairer the scheduler, the less the fluctuations. We, therefore, would expect to measure higher QoE values for fairer schedulers, something that we are going to investigate and quantify next.

Table 1: Basic input simulation parameters.

\begin{tabular}{|c|c|}
\hline Parameter & Values \\
\hline \hline Macro-cell radius & $0.5 \mathrm{~km}$ \\
\hline eNB & 1 eNB, omnidirectional \\
\hline eNB TX power & $43 \mathrm{dBm}$ \\
\hline Number of users & Configurable \\
\hline Distribution of users & Uniform \\
\hline Traffic load per user & Full buffer \\
\hline Duplex mode & FDD (focus on downlink) \\
\hline Channel bandwidth & $5 \mathrm{MHz}$ \\
\hline Number of resource blocks & 25 \\
\hline Flow duration & $30 \mathrm{~s}$ \\
\hline Scheduler implementations & {$[11]$} \\
\hline QoE estimation models & LTD, AREA, constant \\
\hline QoE formula & $0.45 * \ln (A D T)+2.48$ \\
\hline
\end{tabular}

\section{B. QoE-specific analysis}

In this section, we compare the aforementioned schedulers in terms of a) the average and b) effective average download throughput that they achieve (ADT and EADT, respectively), c) the average QoE that they offer, d) the distribution of MOS scores for all users in the cell, e) their fairness, and f) the QoE model used. For the QoE estimations, we have used the LTD and AREA models, as well as a model that ignores fluctuations, namely assumes constant throughput (which is the currently standard approach). The collected results are presented in Figure 3. 
First of all, comparing Figures (3a) and (3b) we observe that the average throughput is much higher than the effective average download throughput, while the latter better correlates to the real user QoE, i.e. to the MOS values in Figure (3c). Moreover, the Proportional Fair and Resource Fair schedulers provide better QoE than the Maximum Throughput scheduler. This observation actually reveals the significance of designing and evaluating a network on a QoE- rather than a QoS-basis, and more specifically, in the context of this paper, it emphasizes the need for scheduling on a QoE-basis.

Similarly, what is validated from Figures (3c)-(3e) is that those schedulers that perform better in terms of QoE are also the fairest ones. This is revealed by the Jain's fairness index presented in Figure (3e) (this index takes values 0..1, where 1 represents the fairest), but also by the empirical CDF plots in Figure (3d). Through the CDF plots it is depicted that fairer schedulers do not cause a high deviation among the MOS scores of different users in the cell (so CDFs are steeper). This is an indication of network stability and consistency in the radio scheduling decisions, which is only achieved by fairer schedulers (i.e. Proportional Fair and Resource Fair).

Finally, examining Figure (3f) in terms of the different QoE models implemented, we observe that those models that consider a mean constant throughput actually overestimate the experience of the users. LTD or AREA models give QoE estimations closer to reality (see section II), since they account for the impact of throughput fluctuations on QoE.

In the next section, we are going to take advantage of the previous conclusions and propose a more consistent, fluctuations-avoiding scheduler.

\section{Designing a Consistent Scheduler}

In the previous sections, the impact of throughput fluctuations on QoE has been revealed and it has been shown that the fluctuations' effect can be indirectly moderated at some extent by using fairer schedulers. However, a more efficient way to achieve that is to design new schedulers that explicitly mitigate these fluctuations.

We therefore propose a fluctuations-aware, consistent scheduler. This scheduler takes into account the evolution of the achieved throughput over time (per user) and the impact of this evolution on the end-user QoE, an aspect not currently addressed by any state of the art schedulers.

The fluctuations' effect may be moderated, i.e. smoothed out, by introducing a new metric that tries to capture and mitigate the magnitude and occurrence of fluctuations. The purpose of this metric is to quantify the gap between the average throughput value over a time window in the past (say $\overline{R^{\imath}}(t-$ 1)) and the expected data rate for the current time interval for each user (the sum of all $d_{k}^{i}(t)$ ). The goal is to minimize this gap, namely to minimize the amplitude of the resulting fluctuations. The larger this amplitude, the less the favoring of giving resource $k$ to user $i$. Since a decision needs to be taken jointly for all users and for all the available resources, user $j$ will be allocated with $k$ only if:

$$
m_{j, k}=\max _{i}\left\{m_{i, k-f l u c t}\right\} \quad \forall i=1 . . N, k=1 . . K
$$

Overall a complex optimization problem needs to be solved, with the objective to find the minimum number of resources per user that minimize this user's deviation from his past average throughput. The optimal solution will provide the best possible combinations of resources that minimize the fluctuations for all users at the same TTI. However, in order to find a solution that works in real-time (sub-optimal though), we introduce the following metric:

$$
m_{i, k-f l u c t}=\frac{1}{\overline{R^{l}}(t-1)-r^{i}(t)-d_{k}^{i}(t)}
$$

where:

$$
\overline{R^{\imath}}(t-1)=\frac{\sum_{\tau=t-1-W}^{\tau=t-1} r^{i}(\tau)}{W}
$$

and $W$ is the window length over which the average throughput is estimated, while $r^{i}(t)$ and $d_{k}^{i}(t)$ have the same meaning as for the state of the art schedulers.

The way this scheduler works is depicted in Figure 4 (as an abstract example). Say there are three users in a cell competing for a total of six available resources during one TTI (also known as resource blocks). Each resource block will result in a different data rate when allocated to a different user, subject to the user's channel quality. $\overline{R^{1}}$ to $\overline{R^{3}}$ are the past average throughput values per user, which the scheduler tries to maintain in order to avoid fluctuations. Therefore, the decisions will be as shown in Figure 4. Each decision is taken per resource block, and it is based on minimizing the gap between $\overline{R^{\imath}}$ and the data rate progressively achieved per user in the current scheduling interval. (The achieved data rate, $r$, is progressively increased every time a user gets another resource block in the current TTI).

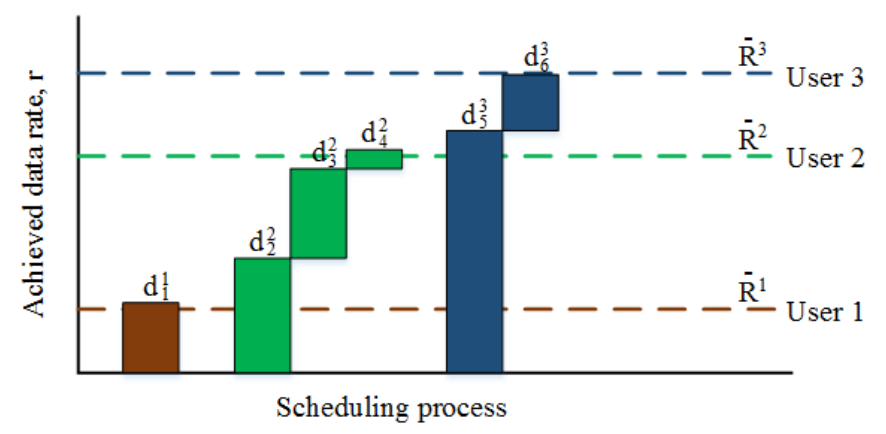

Figure 4: Scheduling logic of the proposed consistent scheduler.

\section{EVALUATION STUDY}

For the purposes of this evaluation study, we implement the proposed scheduling algorithm into the LTE-A simulator of [10]. In the first evaluation study, we aim to prove the concept of the proposed metric for a specific user in the cell.

The results are shown in Figure 5, where we can visualize the successful fluctuations' mitigation. A comparison is done with the Proportional Fair and Resource Fair schedulers, while for the Maximum Throughput scheduler the differences are much higher. Note that we have used the PF and RF schedulers for the 100 first TTIs (Figures 5a and b respectively), after which the proposed fluctuations-avoiding scheduler is activated $(W=100 \mathrm{TTI})$. 

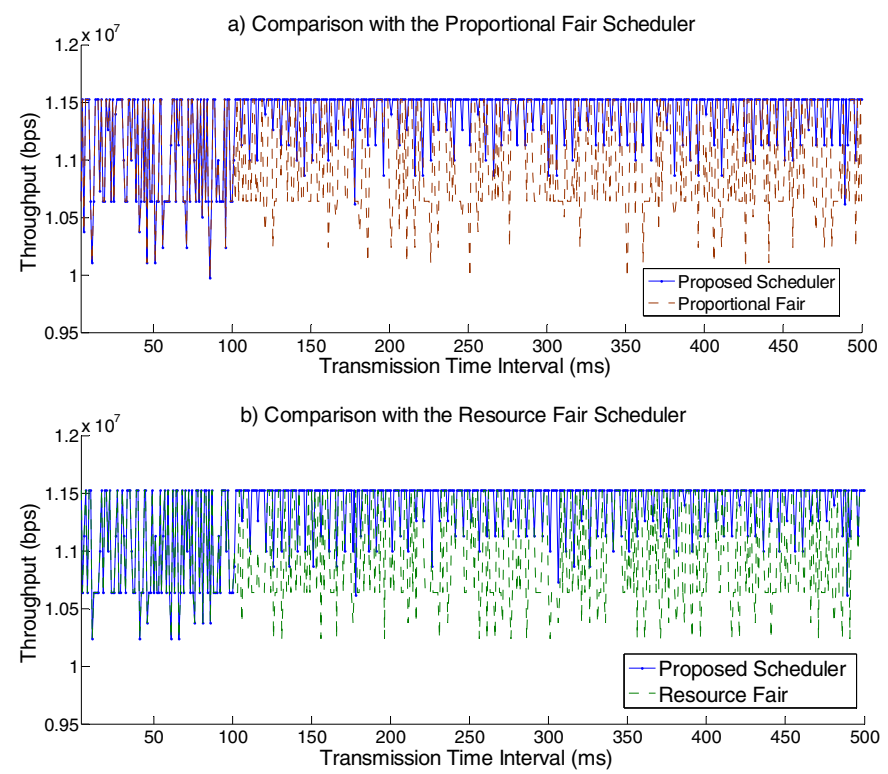

Figure 5: Proof of concept of the proposed scheduler.

Next, we compare the CDF of the proposed scheduler with the state of the art schedulers, for the case of 20 users uniformly distributed in the cell. The results are presented in Figure 6. We can observe that the proposed scheduler (blue line) a) is very fair, as shown by the steepness of the CDF, b) that the achieved minimum MOS values are higher than for the other schedulers (CDF shifted to the right) while c) the larger MOS values are comparable to the other schedulers. This behavior is explained by the fact that the resource allocation procedure of the proposed scheduler is greedy in some sense. By trying to minimize the gap between the average throughput values and the potentially achieved data rates jointly for all the users, eventually this scheduler manages to first satisfy the lowthroughput users. This happens, because the lower the average $\overline{R^{l}}(t-1)$, the lower the difference to the achieved data rate $d_{k}^{i}(t)$ and thus the higher the scheduling priority. However, the low-throughput users do not necessarily take the "best" resource blocks, and therefore higher-throughput users are also served well.

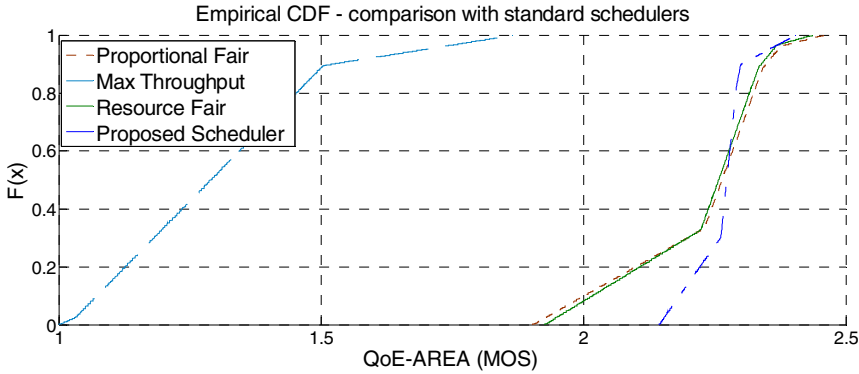

Figure 6: AREA-MOS CDF for the $\boldsymbol{m}_{i, k-\text { fluct }}$ metric.

\section{CONCLUSIONS}

One aspect that has only recently been acknowledged regards the impact of throughput fluctuations on the perceived enduser QoE. This is the reason why "consistency" is an aspect lacking appropriate attention in current state of the art radio schedulers. This paper tries to cover this gap by explaining the meaning and significance of taking consistent radio scheduling decisions, proposing in parallel this novel research direction for future works.

With this in mind, we have evaluated exemplary scheduling algorithms in a realistic LTE-A network simulator. We have reached the conclusion that fairness inherently favors consistency, which is a valuable attribute among different users, but also regarding a single user. On the one hand, consistency among different users is desirable so that the expectations of users co-located in the same cell are similar. On the other hand, consistency over time for a single user is also essential, as it has been revealed by the discussed studies that map per-user throughput fluctuations to QoE. In this paper, we have validated this conclusion by demonstrating that fairer schedulers outperform maximum throughput ones in terms of QoE, as can be measured by proper KPIs.

Nevertheless, these fair exemplary schedulers only indirectly account for the per-user fluctuations. Having identified this deficiency, we have proposed a novel fluctuations-avoiding scheduler that explicitly smooths throughput fluctuations. The measured achieved QoE improvements demonstrate the potential of this scheduler as well as the significance of research towards that direction. Therefore, future work is required in order to design more sophisticated fluctuationsaware schedulers that optimize the decision making process, considering in parallel real-time constraints.

\section{ACKNOWLEDGMENTS}

This work has been funded by the EC under the auspices of the FP7-PEOPLE MITN-CROSSFIRE project (grant 317126) and the H2020-MSCA-RISE CASPER project (grant 645393).

\section{REFERENCES}

[1] NGMN 5G Whitepaper, Version 1.0, March 2015

[2] P. Casas, et al., "On the quest for new KPIs in mobile networks: The impact of throughput fluctuations on QoE," in IEEE Workshop on Quality of Experience-based Management for Future Internet Applications and Services (QoE-FI), pp. 1705-1710, 2015, London.

[3] T. Hossfeld, et al., "Identifying QoE optimal adaptation of HTTP adaptive streaming based on subjective studies," Computer Networks, vol. 81, pp. 320 - 332, Apr. 2015.

[4] G. Tian, and Y. Liu, "Towards agile and smooth video adaptation in dynamic HTTP streaming," in International Conference on Emerging Networking Experiments and Technologies (CoNEXT), pp. 109-120, 2012, Nice.

[5] E. Jammeh, et al., "Quality of Experience (QoE) driven adaptation scheme for voice/video over IP," Telecommun. Syst., vol. 49, no. 1, pp. 99-111, Jan. 2012.

[6] G. de Veciana, et al., "Rate adaptation and admission control for video transmission with subjective quality constraints," IEEE J. Sel. Top. Signal Process., vol. 9, no. 1, pp. 22-36, Feb. 2015.

[7] S. Thakolsri, et al., "QoE-Based Cross-Layer Optimization of Wireless Video with Unperceivable Temporal Video Quality Fluctuation," in IEEE International Conference on Communications (ICC), pp. 1-6, 2011, Kyoto.

[8] A. Sackl, et al., "Quantifying the impact of network bandwidth fluctuations and outages on Web QoE," in IEEE International Workshop on Quality of Multimedia Experience (QoMEX), 2015, Costa Navarino.

[9] F. Capozzi, et al., "Downlink packet scheduling in LTE cellular networks: Key design issues and a survey," IEEE Commun. Surv. Tutorials, vol. 15, no. 2, pp. 678-700, Jan. 2013.

[10] M. Taranetz, et al., "Runtime precoding: Enabling multipoint transmission in LTE-Advanced system-level simulations," IEEE Access, vol. 3, pp. 725-736, Jun. 2015.

[11] S. Schwarz, et al., "Low complexity approximate maximum throughput scheduling for LTE," in Asilomar Conference on Signals, Systems and Computers, pp. 1563-1569, 2010, Pacific Grove, CA. 